\title{
Pharyngeal Mucositis, CTCAE
}

National Cancer Institute

\section{Source}

National Cancer Institute. Pharyngeal Mucositis, CT CAE. NCI Thesaurus. Code C143756.

A disorder characterized by ulceration or inflammation involving the mucous membrane of the pharynx. 